\title{
Role of Spin in Quasiparticle Interference
}

\author{
J. I. Pascual, ${ }^{1,2}$ G. Bihlmayer, ${ }^{5}$ Yu. M. Koroteev, ${ }^{3,4}$ H.-P. Rust, ${ }^{2}$ G. Ceballos, ${ }^{2}$ M. Hansmann, ${ }^{2}$ K. Horn, ${ }^{2}$ E. V. Chulkov, ${ }^{3,6}$ \\ S. Blügel, ${ }^{5}$ P. M. Echenique, ${ }^{3,6}$ and Ph. Hofmann ${ }^{7, *}$ \\ ${ }^{1}$ Institut für Experimentalphysik, Freie Universität Berlin, 14195 Berlin, Germany \\ ${ }^{2}$ Fritz-Haber-Institut der Max-Planck-Gesellschaft, 14195 Berlin, Germany \\ ${ }^{3}$ Donostia International Physics Center (DIPC), 20018 San Sebastián/Donostia, Basque Country, Spain \\ ${ }^{4}$ Institute of Strength Physics and Materials Science, Russian Academy of Sciences, 634021, Tomsk, Russia \\ ${ }^{5}$ Institut für Festkörperforschung, Forschungszentrum Jülich, 52425 Jülich, Germany \\ ${ }^{6}$ Departamento de Física de Materiales and Centro Mixto CSIC-UPV/EHU, Facultad de Ciencias Químicas, UPV/EHU, \\ 20080 San Sebastián/Donostia, Basque Country, Spain \\ ${ }^{7}$ Institute for Storage Ring Facilities, University of Aarhus, 8000 Aarhus C, Denmark
}

(Received 21 July 2004; published 2 November 2004)

\begin{abstract}
Quasiparticle interference patterns measured by scanning tunneling microscopy can be used to study the local electronic structure of metal surfaces and high-temperature superconductors. Here, we show that even in nonmagnetic systems the spin of the quasiparticles can have a profound effect on the interference patterns. On $\mathrm{Bi}(110)$, where the surface state bands are not spin degenerate, the patterns are not related to the dispersion of the electronic states in a simple way. In fact, the features which are expected for the spin-independent situation are absent and the observed interference patterns can be interpreted only by taking spin-conserving scattering events into account.
\end{abstract}

DOI: 10.1103/PhysRevLett.93.196802

PACS numbers: 73.20.At, 68.37.Ef, 71.18.+y, 72.10.Fk

The coupling of spin and orbital angular momenta leads to spin-dependent phenomena even in nonmagnetic materials [1]. For example, spin-orbit coupling (SOC) can remove the spin degeneracy of electronic bands and split them. This effect [2] can be used to inject spin-polarized currents into spintronic devices from a nonmagnetic quantum well structure [3]. On metal surfaces a strong SOC can profoundly change the Fermi surface, screening, and electron dynamics [4]. Despite the variety of manifestations of the SOC effect in condensed matter, some fundamental questions remain unresolved.

A particularly interesting question is how the spin affects the so-called quasiparticle interference (QI) [5]. The term QI is used to describe periodic modulations in the local density of states on surfaces which are caused by the interference of electrons, or quasiparticles, in the vicinity of defects. These modulations are closely related to the Friedel oscillations in the Lindhard picture of screening in metals. One possible approach to investigate the QI is the observation of oscillatory patterns in the local density of states with a scanning tunneling microscope (STM) [6]. Especially, a Fourier transformation (FT) of the STM conductance images can give valuable information about the electronic structure and Fermi surface of quasi-two-dimensional systems [7-10]. The analysis of QI patterns by a STM has found a wide range of applications, such as probing the electronic structure of nanoscale objects [11], contacting of molecular wires [12] as well as measuring the superconducting energy gap function, and possible local ordering on high-temperature superconductors $[10,13,14]$. However, only a little atten- tion has so far been paid to the fact that the quasiparticles can also have a spin.

Here, we show that the quasiparticle spin can have dramatic effects on the interference patterns. In particular, it completely suppresses the interference events associated with the Fermi surface which are usually observed on metal surfaces[6-9]. Instead, the patterns are dominated by spin-conserving processes. We use the (110) surface of Bi to illustrate this. $\mathrm{Bi}(110)$ is ideal for such experiments for two reasons: First, it can be viewed as a two-dimensional metal because it supports a number of surface states crossing the Fermi level $\left(E_{F}\right)$ [15], whereas the bulk density of states at $E_{F}$ is negligible for our purposes. Second, the spin-orbit (SO) interaction leads to a strong splitting of the surface state bands [4], resulting in bands which are nondegenerate with respect to spin.

The $\operatorname{Bi}(110)$ surface was cleaned by sputtering and annealing. STM data were taken using a low-temperature microscope [16] at $5 \mathrm{~K}$. The calculations have been performed by using the full-potential linearized augmented plane wave method in film geometry as implemented in the FLEUR program and local density approximation for the description of exchange-correlation potential. Technical details can be found in Ref. [4].

In order to lay the ground for an interference pattern analysis on $\mathrm{Bi}(110)$, consider its electronic structure as determined by angle-resolved photoemission spectroscopy (ARPES) and first-principles calculations [Fig. 1(a)]. The agreement between ARPES and calculations is excellent, but only if the SO interaction is taken into 


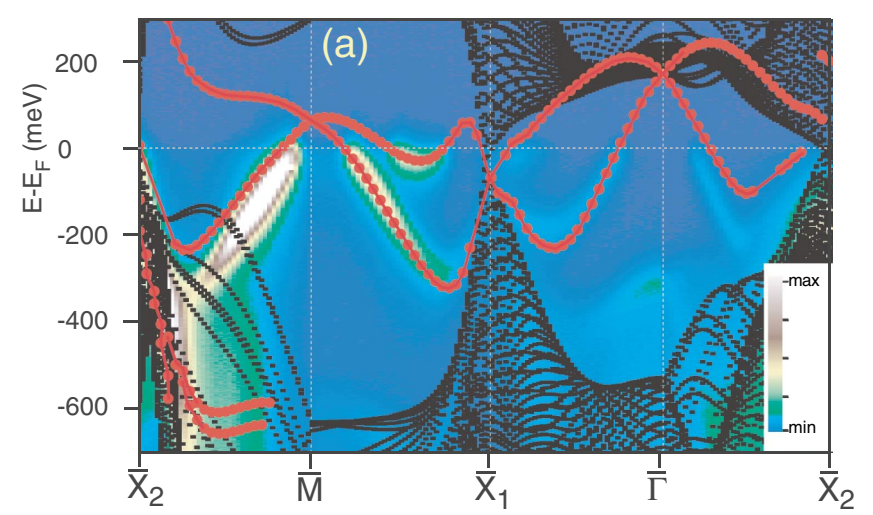

(b)

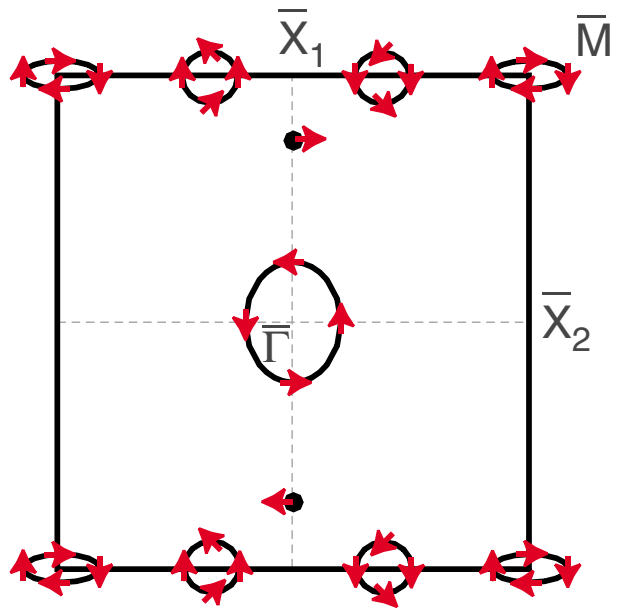

FIG. 1 (color). (a) Surface electronic structure of Bi(110). The color scale plot is the linear photoemission intensity measured by ARPES, published in Ref. [15]; the red markers are the result of our ab initio calculation (see Ref. [4]). (b) Sketch of the SBZ and the Fermi surface with an indication of the approximate spin direction.

account in the latter. At the surface, the SO interaction lifts the Kramers degeneracy and all the calculated bands are nondegenerate; i.e., they contain only one spin per band and $k$ (momentum) point. Only at some special points of the surface Brillouin zone (SBZ), such as $\bar{\Gamma}$ and $\bar{M}$, symmetry forces the two spin-split bands to be degenerate [4]. The large splitting results in a Fermi surface (FS) with four distinct, non-spin-degenerate elements [see Fig. 1(b)]. There are two hole pockets around the $\bar{\Gamma}$ and $\bar{M}$ points, a shallow electron pocket centered on the $\bar{M}-\bar{X}_{1}$ line and a very small feature along $\bar{\Gamma}-\bar{X}_{1}$ which can be viewed as a single point for the present purpose. The approximate spin directions on the Fermi surface are indicated by red arrows. In the most simple picture, the spin is oriented in the plane of the surface and perpendicular to the propagation direction. This is exactly true for a two-dimensional gas of free electrons where the SO interaction can be treated by adding a so-called Rashba term to the Hamiltonian [2]. Here, the spin directions can be worked out by inspecting the band dispersion in Fig. 1(a). For example, the hole pocket states near $\bar{\Gamma}$ and
$\bar{M}$ are formed by the lower branch of the SO split bands. Therefore, the relative orientation between the twodimensional momentum $\vec{k}$ and the spin has to be the same for both states. Hence, the indicated direction of the spin has to rotate counterclockwise and clockwise around the $\bar{\Gamma}$ and $\bar{M}$ contours, respectively. For the electron pocket along $\bar{M}-\bar{X}_{1}$, the spin polarization merely "wiggles" around the preferred direction because of the need to take into account the symmetry requirement imposed by the crossing of the SBZ boundary.

In a simple picture, a quasiparticle interference pattern near $E_{F}$ arises because an electron with wave vector $\vec{k}_{F}$ encounters a defect, such as an impurity or a step edge, and is reflected into a state with wave vector $-\vec{k}_{F}$. The interference of incoming and reflected waves gives rise to a modulation in the local density of states with a periodicity of $2 \vec{k}_{F}$, i.e., with the vector connecting the two states. A Fourier transform of STM differential conductance $(d I / d V)$ maps near $E_{F}$, therefore, shows an image of the two-dimensional Fermi surface with high intensities at points with $2 \vec{k}_{F}$ [7-9]. On high-temperature superconductors, similar interference patterns are observed but the intensities in the FT maps are strongly influenced by the density of the states causing the scattering events [10].

The spin map from Fig. 1(b) raises questions about the possible quantum interference phenomena in the present case because states with $\vec{k}_{F}$ and $-\vec{k}_{F}$ have opposite spin directions. Therefore, all of the interference processes building up the FS topology in the FT maps should be forbidden [5].

In order to test this hypothesis, we now analyze the interference patterns on $\mathrm{Bi}(110)$. Figure 2(a) shows atomically resolved images of the surface topography close to a step on the surface. Bi(110) consists of a bilayerlike structure with an almost square unit cell, containing two atoms [15]. One of the atoms binds to the underlying layer; the other atom presents a dangling bond. The STM resolves only this latter atom.

The energy-dependent quasiparticle interference can be studied by $d I / d V$ maps. Such maps are shown in Figs. 2(b)-2(e) for different bias voltages. For bias voltages below the onset of the surface state bands $(V<$ $-300 \mathrm{mV}$ ), the maps show periodic structures very similar to the topography images. Above the onset $(V>$ $-300 \mathrm{mV}$ ), weak sinelike modulations along $\bar{\Gamma}-\bar{X}_{1}$ and $\bar{\Gamma}-\bar{X}_{2}$ become clearly visible. Finally, $d I / d V$ maps close to $E_{F}$ show a pronounced modulation along $\bar{\Gamma}-\bar{X}_{1}$. Figure 2(f) compares the strength of the lattice-periodic modulations as a function of the bias voltage close to $E_{F}$. It displays the intensity of the corresponding spots in the FT of a set of conductance images. It is evident that an enhancement of the modulation only along the $\bar{\Gamma}-\bar{X}_{1}$ direction sets in for bias voltages above $-100 \mathrm{meV}$. 


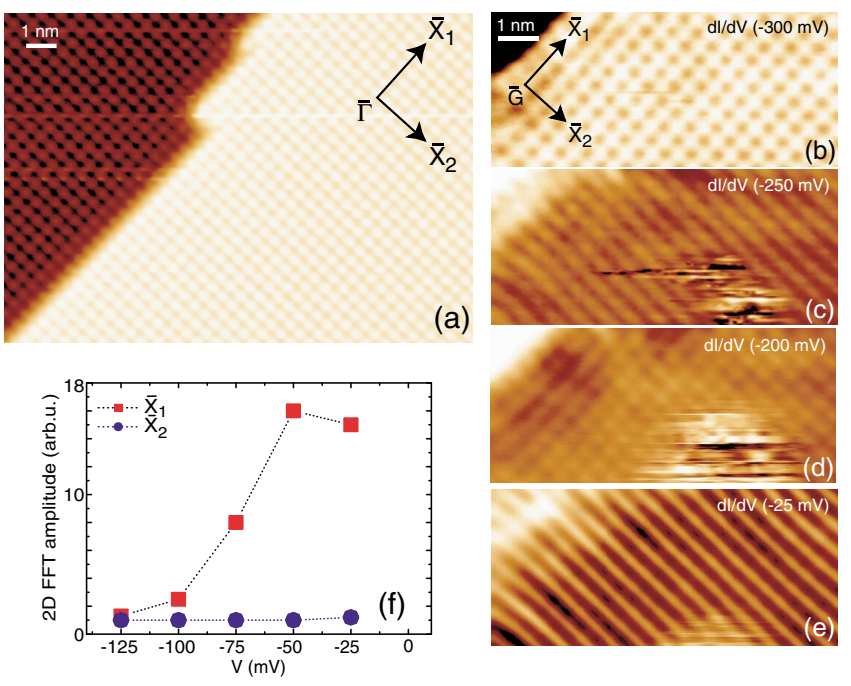

FIG. 2 (color). (a) Atomically resolved constant current images of $\mathrm{Bi}(110)$ near a step $(V=-205 \mathrm{mV} ; I=1 \mathrm{nA})$; (b)(e) conductance images taken at different bias voltages $(I=$ $1 \mathrm{nA}$ ); (f) intensity of the lattice spots in the FT conductance images as a function of bias voltage.

It is important to realize that the lattice spots are a genuine spectroscopic feature and not merely caused by the geometric structure of the surface. Their origin is related to the interference of Bloch wave-type surface states [17-19]. In the present context, they have a particular importance because some umklapp-type interference processes, involving a reciprocal lattice vector $\vec{g}$, are not forbidden by spin. For example, interference between $\left(\vec{k}_{F}, \leftarrow\right)$ and $\left(\vec{k}_{F}+\vec{g}, \leftarrow\right)$ is always permitted, while interference between $\left(\vec{k}_{F}, \leftarrow\right)$ and $\left(-\vec{k}_{F}, \rightarrow\right)$ is not. On the basis of this, it is easy to interpret the strong change of the $\bar{X}_{1}$ lattice spot intensity in Fig. 2(f) around $-75 \mathrm{meV}$. An inspection of the band structure in Fig. 1(a) reveals that the bottom of the shallow electron pocket along $\bar{X}_{1}-\bar{M}$ happens to be at this energy. For lower energies, the electron pocket can not contribute to any interference events and the intensity of the $\bar{X}_{1}$ lattice spots drops.

To study the QI away from the lattice spots, Figs. 3(a) and 3(b) show a typical $d I / d V$ image taken at $V=$ $+40 \mathrm{mV}$ together with its Fourier transformation. The bias voltage is sufficiently small to allow a direct comparison of the observed interference features with the Fermi surface sketched in Fig. 1(b). Figure 3(c) shows a schematic drawing of the FT map (colored features) together with the expected interference pattern for a spin-independent situation (gray dashed lines). It is evident that the latter is totally absent from the FT map, in accordance with the simple picture outlined above. Instead, a number of other features are observed in addition to the lattice spots.

The first of these, labeled $A$ in Fig. 3(c), is a fuzzy ellipse around the origin $\bar{\Gamma}$, which can be also detected around the $\bar{X}_{1}$ points. The longer radius is of the order of
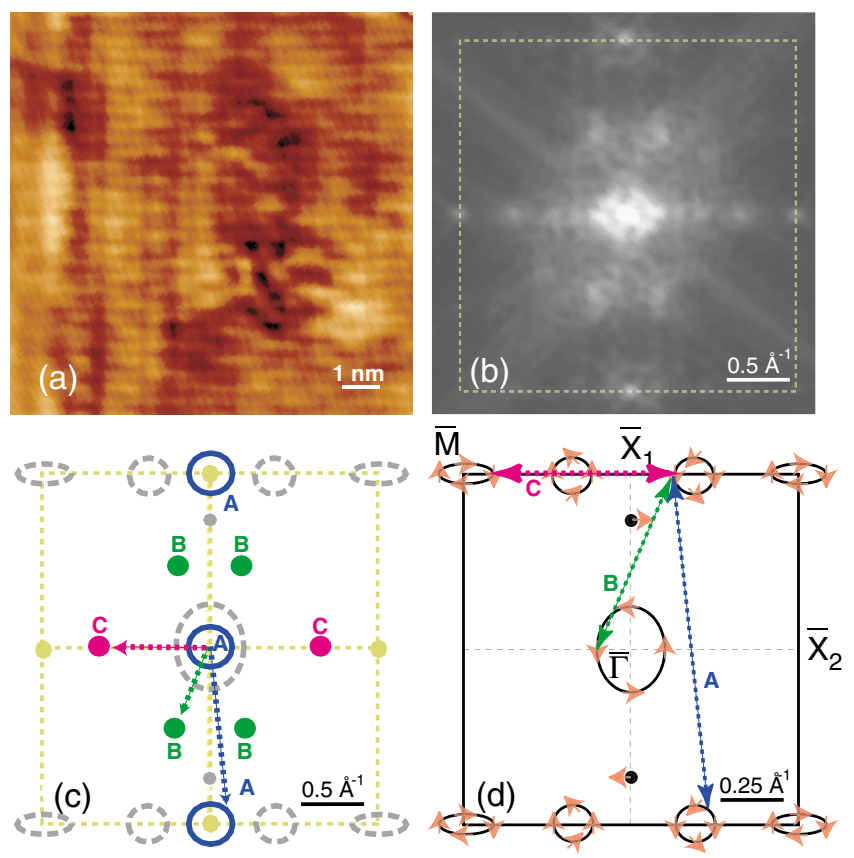

FIG. 3 (color). (a) Differential conductance map $(V=$ $40 \mathrm{mV} ; I=1 \mathrm{nA}$ ) and (b) its FT map. (c) Schematic drawing of the FT map. The dashed gray lines are the modulations which would be expected for quasiparticle interference from a Fermi surface in Fig. 1(b) if the spin was not important. The features $A, B$, and $C$ are the actually observed non-latticeperiodic structures. The yellow markers represent the lattice spots. (d) Illustration of the spin-conserving scattering events causing features $A, B$, and $C$. The dashed scattering vectors have to be translated to the origin and scaled down by a factor of 2 to yield the features in (c).

$0.2 \AA^{-1}$. The second feature, labeled $B$ in the figure, consists of four spots at a distance of 0.62 and $0.26 \AA^{-1}$ from $\bar{\Gamma}$ in the $\bar{\Gamma}-\bar{X}_{1}$ and $\bar{\Gamma}-\bar{X}_{2}$ directions, respectively. Features $C$ are two spots on either side of the center, lying in the $\bar{\Gamma}-\bar{X}_{2}$ direction. Their distance from $\bar{\Gamma}$ is $0.9 \AA^{-1}$.

We can explain these additional features by considering only the scattering processes which conserve the spin. Some of these are indicated by dashed lines in Fig. 3(d). They lead to interference patterns with a periodicity of $\vec{q}=\vec{k}_{\text {final }}-\vec{k}_{\text {initial }}$. The resulting $\vec{q}$ 's are also shown in Fig. 3(c), taking the scaling difference of 3(c) and 3(d) into account. Using these vectors, we can identify the $B$ and $C$ features as caused by interference between the electron pockets along the $\bar{M}-\bar{X}_{1}$ direction and hole pocket states around $\bar{\Gamma}$ and $\bar{M}$, respectively. For the $A$ structures the most likely origins are scattering processes between states in the electron pockets along $\bar{M}-\bar{X}_{1}$. Interference due to these scattering processes would be expected to show up as elliptical features around the $\bar{X}_{1}$ lattice spots only. It is well known, however, that shifted replicas of such features can also be found around the origin. These shifted replicas can be much more intense 
than the originals around the higher order lattice spots, depending on the character of the surface wave function [18].

Our findings show that taking the spin into account can be essential for the understanding of QI. Indeed, the interference patterns on $\mathrm{Bi}(110)$ cannot be directly related to the Fermi surface topology as it happens for spindegenerate bands [9], nor can they be understood in terms of wave-vectors connecting points of a high density of states $[10,13]$. The absence of these "normal" interference patterns can be explained by taking the spin direction into account. Instead, we find strong, energy-dependent lattice spots and spectroscopic signatures of spinconserving interference patterns.

The formation of standing electron waves in a situation where the spin plays a role was initially discussed for the surface states on $\mathrm{Au}(111)$. As was found by ARPES and confirmed by first-principles calculations, this surface state band is split by the SO interaction [20,21]. The splitting leads to a lift of the spin degeneracy and changes the dispersion from a simple parabolic band to two slightly shifted parabolas. The Fermi surface is turned from a circle into two concentric circles of nearly the same radius. An analysis of QI close to the Fermi level, however, revealed only one circle [22] and this was explained by the fact that quasiparticles with opposite spin cannot give rise to observable interference patterns [5]. In this case, the spin seems to induce only a small error in the Fermi surface determined by QI. Our results show, however, that the observed QI pattern can be profoundly changed in the case of strong spin-orbit splitting and an interpretation of our data as an image of the Fermi surface would be completely misleading.

The consequences from the present work are twofold: First, it is obviously essential to take the spin into account when interpreting quasiparticle interference. This will be especially relevant for complex system with low symmetry where the spin character of the states is not known a priori. More importantly, our results open entirely new possibilities by showing that the spin character of the quasiparticles is reflected in the screening of defects on the surface and can be probed by a nonmagnetic STM. The combination of known Fermi surface and observed interference pattern gives direct information about the relative spin of the quasiparticles at the Fermi surface. Such information can be important, for example, when discussing different pairing mechanisms leading to superconductivity or the possibility of spin-charge separation.

This work was partially supported by the UPV/EHU, Spanish MCyT and by the Danish National Science Foundation.
*Electronic address: philip@phys.au.dk Electronic address: http://www.phys.au.dk/ philip/

[1] R. Winkler, Spin-Orbit Coupling Effects in TwoDimensional Electron and Hole Systems, Springer Tracts in Modern Physics Vol. 191 (Springer-Verlag, Berlin, 2003).

[2] É. I. Rashba, Sov. Phys. Solid State 2, 1109 (1960).

[3] T. Koga, J. Nitta, H. Takayanagi, and S. Datta, Phys. Rev. Lett. 88, 126601 (2002).

[4] Yu. M. Koroteev, G. Bihlmayer, J. E. Gayone, E. V. Chulkov, S. Blügel, P. M. Echenique, and Ph. Hofmann, Phys. Rev. Lett. 93, 046403 (2004).

[5] L. Petersen and P. Hedegård, Surf. Sci. 459, 49 (2000).

[6] M. F. Crommie, C. P. Lutz, and M. Eigler, Nature (London) 363, 524 (1993).

[7] P.T. Sprunger, L. Petersen, E.W. Plummer, E. Lægsgaard, and F. Besenbacher, Science 275, 1764 (1997).

[8] Ph. Hofmann, B. G. Briner, M. Doering, H.-P. Rust, E.W. Plummer, and A. M. Bradshaw, Phys. Rev. Lett. 79, 265 (1997).

[9] L. Petersen, P.T. Sprunger, Ph. Hofmann, E. Lægsgaard, B. G. Briner, M. Doering, H.-P. Rust, A. M. Bradshaw, F. Besenbacher, and E.W. Plummer, Phys. Rev. B 57, R6858 (1998).

[10] K. McElroy, R.W. Simmonds, J. E. Hoffman, D.-H. Lee, J. Orenstein, H. Eisaki, and J. C. Davis, Nature (London) 422, 592 (2003).

[11] L. Diekhöner, M. A. Schneider, A. N. Baranov, V.S. Stepanyuk, P. Bruno, and K. Kern, Phys. Rev. Lett. 90, 236801 (2003).

[12] F. Moresco, L. Gross, M. Alemani, K.-H. Rieder, H. Tang, A. Gourdon, and Ch. Joachim, Phys. Rev. Lett. 91, 036601 (2003).

[13] J. E. Hoffman, K. McElroy, D.-H. Lee, K. M. Lang, H. Eisaki, S. Uchida, and J.C. Davis, Science 297, 1148 (2002).

[14] M. Vershinin, S. Misra, S. Ono, Y. Abe, Y. Ando, and A. Yazdani, Science 303, 1995 (2004).

[15] S. Agergaard, C. Søndergaard, H. Li, M. B. Nielsen, S. V. Hoffmann, Z. Li, and Ph. Hofmann, New J. Phys. 3, 15 (2001).

[16] H.-P. Rust, J. Buisset, E. K. Schweizer, and L. Cramer, Rev. Sci. Instrum. 68, 129 (1997).

[17] B. G. Briner, Ph. Hofmann, M. Doering, H.-P. Rust, E.W. Plummer, and A. M. Bradshaw, Europhys. Lett. 39, 62 (1997).

[18] L. Petersen, B. Schaefer, E. Lægsgaard, I. Stensgaard, and F. Besenbacher, Surf. Sci. 457, 319 (2000).

[19] J. I. Pascual, Z. Song, J. J. Jackiw, K. Horn, and H.-P. Rust, Phys. Rev. B 63, 241103(R) (2001).

[20] S. LaShell, B. A. McDougall, and E. Jensen, Phys. Rev. Lett. 77, 3419 (1996).

[21] G. Nicolay, F. Reinert, S. Hüfner, and P. Blaha, Phys. Rev. B 65, 033407 (2001).

[22] L. Petersen, L. Bürgi, H. Brune, F. Besenbacher, and K. Kern, Surf. Sci. 443, 154 (1999). 